Draft Version April 15, 2018

Preprint typeset using $\mathrm{L}_{\mathrm{E}} \mathrm{EX}$ style emulateapj v. 5/2/11

\title{
OBSERVATIONS OF SUPRA-ARCADE FANS: INSTABILITIES AT THE HEAD OF RECONNECTION JETS
}

\author{
D. E. Innes ${ }^{1,2}$, L.-J. Guo ${ }^{1,2}$, A. BhattacharjeE ${ }^{2,3,4}$, Y.-M. Huang ${ }^{2,3,4}$, And D. Schmit S $^{1,2}$ \\ ${ }^{1}$ Max Planck Institute for Solar System Research, 37077 Göttingen, Germany \\ ${ }^{2}$ Max Planck/Princeton Center for Plasma Physics, Princeton, NJ 08540, USA \\ ${ }^{3}$ Department of Astrophysical Sciences and Princeton Plasma Physics Laboratory, Princeton University, Princeton, NJ 08540, USA and \\ ${ }^{4}$ Center for Integrated Computation and Analysis of Reconnection and Turbulence and Space Science Center, University of New \\ Hampshire, Durham, NH 03824, USA \\ Draft version April 15, 2018
}

\begin{abstract}
Supra-arcade fans are bright, irregular regions of emission that develop during eruptive flares, above flare arcades. The underlying flare arcades are thought to be a consequence of magnetic reconnection along a current sheet in the corona. At the same time, theory predicts plasma jets from the reconnection site which would be extremely difficult to observe directly becuase of their low density. It has been suggested that the dark supra-arcade downflows (SADs) seen falling through supra-arcade fans may be low density jet plasma. The head of a low density jet directed towards higher density plasma would be Rayleigh-Taylor unstable, and lead to the development of rapidly growing low and high density fingers along the interface. Using SDO/AIA $131 \AA$ images, we show details of SADs seen from three different orientations with respect to the flare arcade and current sheet, and highlight features that have been previously unexplained, such as the splitting of SADs at their heads, but are a natural consequence of instabilities above the arcade. Comparison with 3-D magnetohydrodynamic simulations suggests that supra-arcade downflows are the result of secondary instabilities of the Rayleigh-Taylor type in the exhaust of reconnection jets.
\end{abstract}

Subject headings: instabilities - magnetic reconnection - magnetohydrodynamics - Sun:activity Sun:flares

\section{INTRODUCTION}

Supra-arcade fans are diffuse regions of hot, $10 \mathrm{MK}$, plasma seen above flare arcades (Svestka et al. 1998; McKenzie \& Hudson 1999; McKenzie 2000; Hanneman \& Reeves 2014). The supra-arcade fan can extend up to about $150 \mathrm{Mm}$ above the flare arcade and can last for several hours. Often dark, tadpole-shaped structures, known as supra-arcade downflows (SADs), are seen descending through the bright fans, and the top of the arcade appears spiky (McKenzie 2000; Innes et al. 2003b; Asai et al. 2004; Verwichte et al. 2005; Savage \& McKenzie 2011). The SADs which are low density plasma (Innes et al. 2003b; Savage et al. 2012a) are believed to be a consequence of reconnection high in the corona because, among other reasons, they correlate well with bursts of hard X-rays (Asai et al. 2004; Khan et al. 2007). Understanding the cause of SADs could shed light on the reconnection process itself as well as other possible secondary instabilities. One difficulty is that SADs are only visible where there is sufficient contrast with fan emission, so their structure outside the fan is not known. Nevertheless high resolution observations of the interaction between the bright fan plasma and the SADs can possibly discriminate between proposed scenarios.

Supra-arcade fans generally extend along the whole length of the underlying arcade, and are thought to be flat sheet-like structures or the narrow, low density SADs would not show up as dark streaks. When observed above cusp-shaped flare arcades, they have been interpreted as current sheets (Savage et al. 2010; Liu et al.

innes@mps.mpg.de
2013; Liu 2013). These current sheets, seen as rays of increased density and temperature (Ko et al. 2003; Ciaravella \& Ravmond 2008), separate regions of oppositely directed magnetic field above newly formed flare loops. The current sheets are expected to be seen edgeon above arcades seen from one end so that they appear cusp-shaped, and face-on above arcades seen from the side.

SADs are best seen when the current sheet is faceon (Savage et al. 2010; Warren et al. 2011). A few have also been seen below an almost edge-on current sheet (Liu 2013; Liu et al. 2013). In high resolution extreme ultraviolet (EUV) images their appearance changes as the supra-arcade fan develops (see animations in Warren et al. (2011)). Early downflows are often broader and shorter than the more distinctive, tadpoleshaped flows that are seen once the supra-arcade fan has fully developed.

The SADs often appear above and occasionally alongside rapidly retracting loops, known as supraarcade downflow loops (SADLs) with similar velocity (50-400 km s$\left.{ }^{-1}\right)$, acceleration and height (Savage \& McKenzie 2011). SADLs appear bright against the dark corona and SADs are darker than the surrounding supra-arcade fan plasma. It has been noted that contrary to SADs, SADLs are more likely to be seen when the flare arcade is oriented with its axis parallel to the line-of-sight (Savage et al.|2010; Savage \& McKenzie 2011; Warren et al. |2011; Liu et al. 2013; Liu 2013).

Early analyses of SADs concluded that they were evacuated loops seen edge-on; however after studying Solar Dynamics Observatory (SDO) Atmospheric Imaging Assembly (AIA) 193, 131, and $94 \AA$ images, Savage et al. 
(2012b) concluded that they were wakes of retracting loops. Correlation tracking of the surrounding flows has shown that the surrounding plasma is highly turbulent (McKenzie 2013). This is consistent with the SUMER observations of high line-of-sight velocities in the supraarcade fan along the edge of SADs (Innes et al. 2003a; Doschek et al. 2014).

A model, based on 3-D bursty reconnection, demonstrated that teardrop-shaped downflows with trailing low density tails, similar to SADs could be expected from retracting loops (Linton \& Longcope 2006; Guidoni \& Longcope 2011). When the loops descend through the supra-arcade fan, they may drive shocks ahead and rarefactions behind (Scott et al. 2013) travelling with speeds of the order of the local sound speed (350 $\mathrm{km} \mathrm{s}^{-1}$ for $10 \mathrm{MK}$ plasma). The SAD model developed by Costa et al. (2009), Maglione et al. (2011), and Cécere et al. (2012) assumes multiple reconnection sites in which the SADs are a consequence of shocks and rarefactions bouncing back and forth in magnetic structures.

Recently, Cassak et al. (2013) have pointed out that retracting loops or their wakes would be filled too quickly by the surrounding plasma to explain their relatively long lifetime (up to $20 \mathrm{~min}$ ), and propose that the SADs are low density jets produced by multiple reconnection sites along the current sheet. In their scenario, the continual jet flow fills the SAD channel and prevents it from closing; however their model places the voids below, not above, the flare arcade.

An alternative jet-flow model, introduced by Asai et al. (2004), is that, rather than being a consequence of many reconnection jets, the SADs are the result of instabilities at the head of a main jet (TanDokoro \& Fujimoto 2005). The head of a low density jet pushing into high density plasma above the arcade would be Rayleigh-Taylor unstable. There are clear morphological similarities between SADs and the Rayleigh-Taylor plumes and fingers recently identified in high resolution solar observations of quiescent prominences (Berger et al. 2010, 2011; Hillier et al. 2012) and ejected filament plasma falling back through the corona (Innes et al. 2012).

Distinguishing between the various SAD models is a challenge for the observations. In this paper, we look carefully at the early appearance and growth of individual SADs at the top of the arcade fan because this is where models differ. For example, as described in an accompanying paper (Guo et al. 2014), if SADs are caused by instabilities at the interface between the jet and fan plasma, they will exhibit structural evolution at their heads whereas the descending loop (Savage et al. 2012b; Scott et al. 2013) and jet (Cécere et al. 2012; Cassak et al. 2013) models predict relatively stable teardrop-shaped heads. Also growing fan spikes are a natural consequence of the instability model and are themselves expected to be unstable, leading to the appearance of SADs at the top of growing fan spikes. In addition, models differ in the relationship between SADs and SADLs. In the wake model SADLs are expected near the front of all SADs, whereas the Rayleigh-Taylor instability model of Guo et al. (2014) predicts that SADs are low density, reconnected jet plasma that can exist without a SADL at its head. How- ever, as explained in the discussion section, the SADs are threaded by flux rooted in opposite polarities in the photosphere so loops may appear along with SADs if, for example, non-uniformities cause the SAD flux to break up. SADLs have been observed in front of some of the larger SADs as they approached the arcade (Savage et al. 2012b) but the viewing angle for picking up SADs was not optimal and there was considerable confusion along the line-of-sight. We test the SADL-SAD connection by taking advantage of an event that occurred on the disk where we can see both SADs and SADLs with less lineof-sight confusion and with a better orientation for seeing faint SADLs.

\section{OBSERVATIONS}

We investigate SDO/AIA observations of SADs in three eruptive flares with different arcade axis and therefore current-sheet orientations. The flares are shown in Figure1. The top panels show the SDO/AIA images and the panels below, the flares seen from the STEREO Extreme Ultraviolet Imager (EUVI) (Howard et al. 2008). STEREO consists of two identical spacecraft, STEREOA (ahead) and STEREO-B (behind), in heliocentric orbit at $\sim 1$ AU that are increasing their separation from Earth by $\sim 22^{\circ} /$ year. At the time the STEREO spacecraft were between $100^{\circ}$ and $120^{\circ}$ from the Earth-Sun line so SDO limb/disk flares appear as disk/limb flares from STEREO. The arcade axis after the M1.3 flare on 22 October 2011 was perpendicular to the line-of-sight and so the current sheet was seen face-on from SDO (Savage et al. 2012b). The 2012 July 19 M7.7 flare produced a bright, cusp-shaped arcade on the south-west limb. As can be seen from the STEREO image, Figure 1(e), the viewing perspective from SDO was along the arcade axis (Liu et al. 2013; Liu 2013). The M3.2 flare on 2012 January 19 occurred close to disk center, at N28E13, and the arcade which ran north-south was essentially seen from above from SDO.

AIA obtains full Sun images out to $\sim 1.3 \mathrm{R}_{\odot}$ with a spatial resolution of $0.6^{\prime \prime}$ pixel $^{-1}$ and a cadence of $12 \mathrm{~s}$ in 10 EUV and UV channels (Lemen et al. 2012). SADs are visible in all the hot AIA channel images (94, 131, 193, $335 \AA$ ) but are best seen in the $131 \AA$ images. In these images the arcade fan is brightest and most extended. The main contributor to the $131 \AA$ flare emission is Fe XXI which has a formation temperature around 10-12 MK.

\subsection{October 22}

The $131 \AA$ channel images show a large, rapidly evolving supra-arcade fan with a rich variety of SAD structures (see movie_11oct22). Analysis of images from this event led Savage et al. $(2012 \mathrm{~b})$ to conclude that the SADs were not edge-on loops but rather the wakes behind rapidly retracting loops. This is the event where they identified loop-like structures at the heads of some of the SADs as they approached the top of the underlying arcade.

Here we concentrate on the first appearance and evolution of some of the clearest examples of SAD evolution. Figure 2 shows four time frames for four groups of SADs. The corresponding difference images are shown in Figure 3. The first SAD sequence has been picked out because it shows a SAD appearing at the head of a growing fan spike. The growth of the spike, with a velocity 

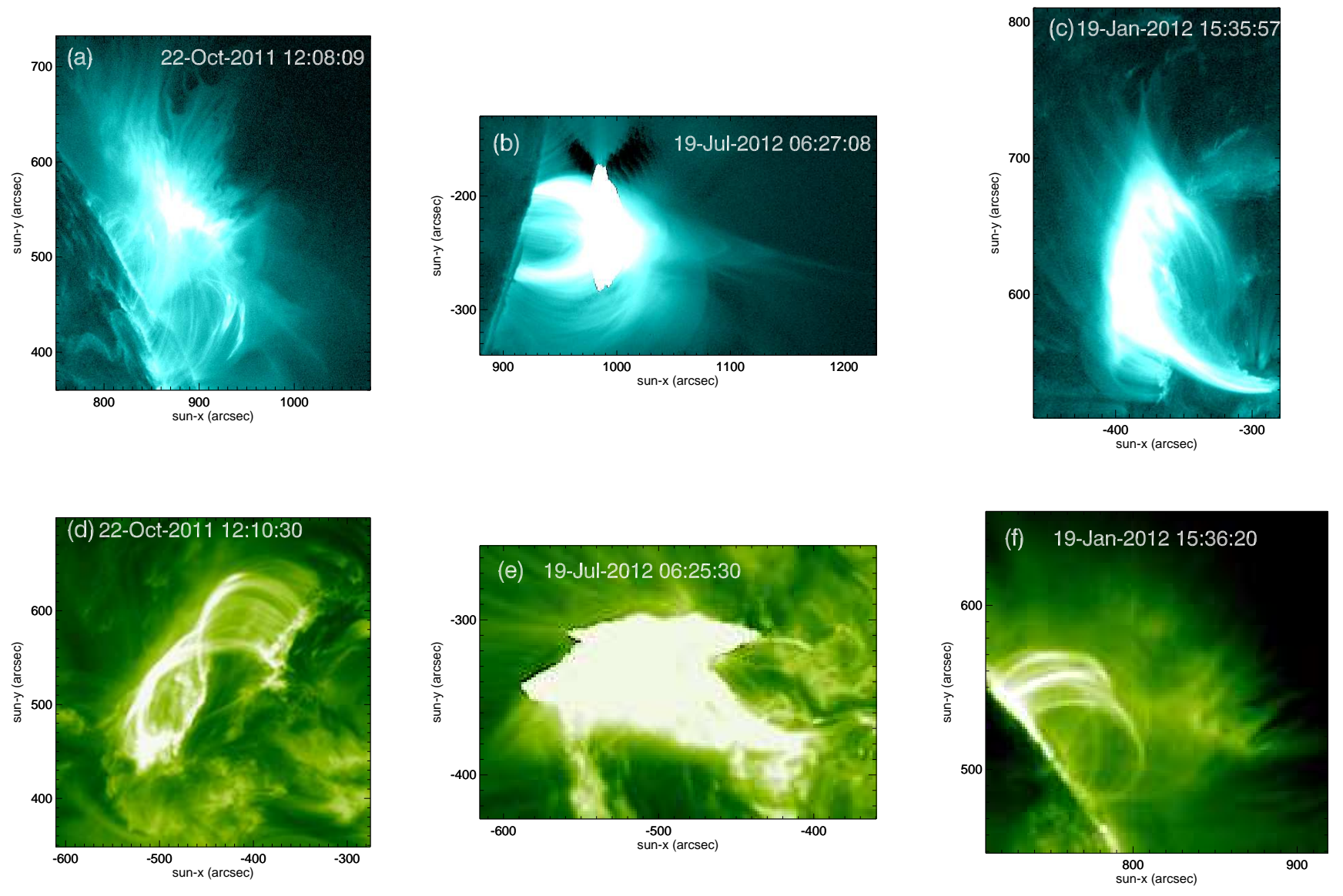

FIG. 1.- (a)-(c) SDO/AIA $131 \AA$ channel images of the flares and supra-arcades fans. (d)-(f) Corresponding STEREO/EUVI $195 \AA$ filter images of the same flares from (d) STEREO-A at $105^{\circ}$, (e) STEREO-A at $120^{\circ}$, (f) STEREO-B at $-113^{\circ}$ Earth ecliptic longitude.

$40 \mathrm{~km} \mathrm{~s}^{-1}$, is clearly visible between frames (a) and (b) in both the intensity and difference images. The SAD, seen first in frame (c), appears at the apex, causing the spike top to split. Both the growing fan spike and the SAD appearance at the top are predicted by the simulations of SADs caused by instabilities in the exhaust of reconnection jets (Guo et al.|2014). An example is shown in the discussion section, Figure [7(b)-(d). The reconnection downflow occurs across the length of the arcade, and the whole interface is unstable. The spikes represent the fastest growing modes. The regions between the spikes are also filled with downflows but these are not as visible because there is less contrast with the fan emission.

The second row shows evolution at the head of one of the wider SADs. The original teardrop-shaped head (f) developed prongs (g) that grow (h) and later separated from the main downflow, creating 3 or 4 tadpolelike SADs (see movie_11oct22). The next example shows prong development at the head of a narrower SAD. In this example, between images (k) and (l), the dark 'tail' remains stationary while the prongs detach and move slowly downward. In this supra-arcade fan, there are several examples of SADs with evolving head structure. We presume it is common, although it has not been reported elsewhere. Further investigation of other well-observed events is required.

The fourth example shows two narrow SADs that remain visible for at least $12 \mathrm{~min}$ although their widths are at most $1.5 \mathrm{Mm}$. Cassak et al. (2013) have sug- gested that if the SADs were wakes, they will rapidly fill with ambient plasma; however, the ambient magnetic field may compensate for the lower plasma pressure in the wake preventing their disappearance. The long lifetime can also be explained if SADs result from quasi-saturated instabilities along an interface between a hot, low-density jet, and denser, cooler arcade plasma because the reconnection flow will keep the SAD from filling with ambient plasma. A more extreme example of a stationary SAD was reported by Hanneman \& Reeves (2014). The one they show is also fork-shaped, and it remained at the same position for about one hour. We also note that the last two narrow SADs simply slowed down and faded in the bright fan plasma. There was no indication of either a loop or enhanced emission at their heads, as has been predicted by some retracting loop models (Scott et al. 2013).

\subsection{July 19}

This bright, cusp-shaped event has been analysed by Liu et al. (2013) and Liu (2013) who have described many aspects related to the dynamics of the bright fan emission, including coronal hard X-ray and microwave sources, fast retracting loops, and SADs. As can be seen in the online movie, movie_12jul19, many SADLs were seen during the initial phase of arcade formation. The earliest SADs, appeared later than the first SADLs. They were analysed by Liu (2013), and are shown again in Figure 4. The first SAD, indicated by a white arrow 


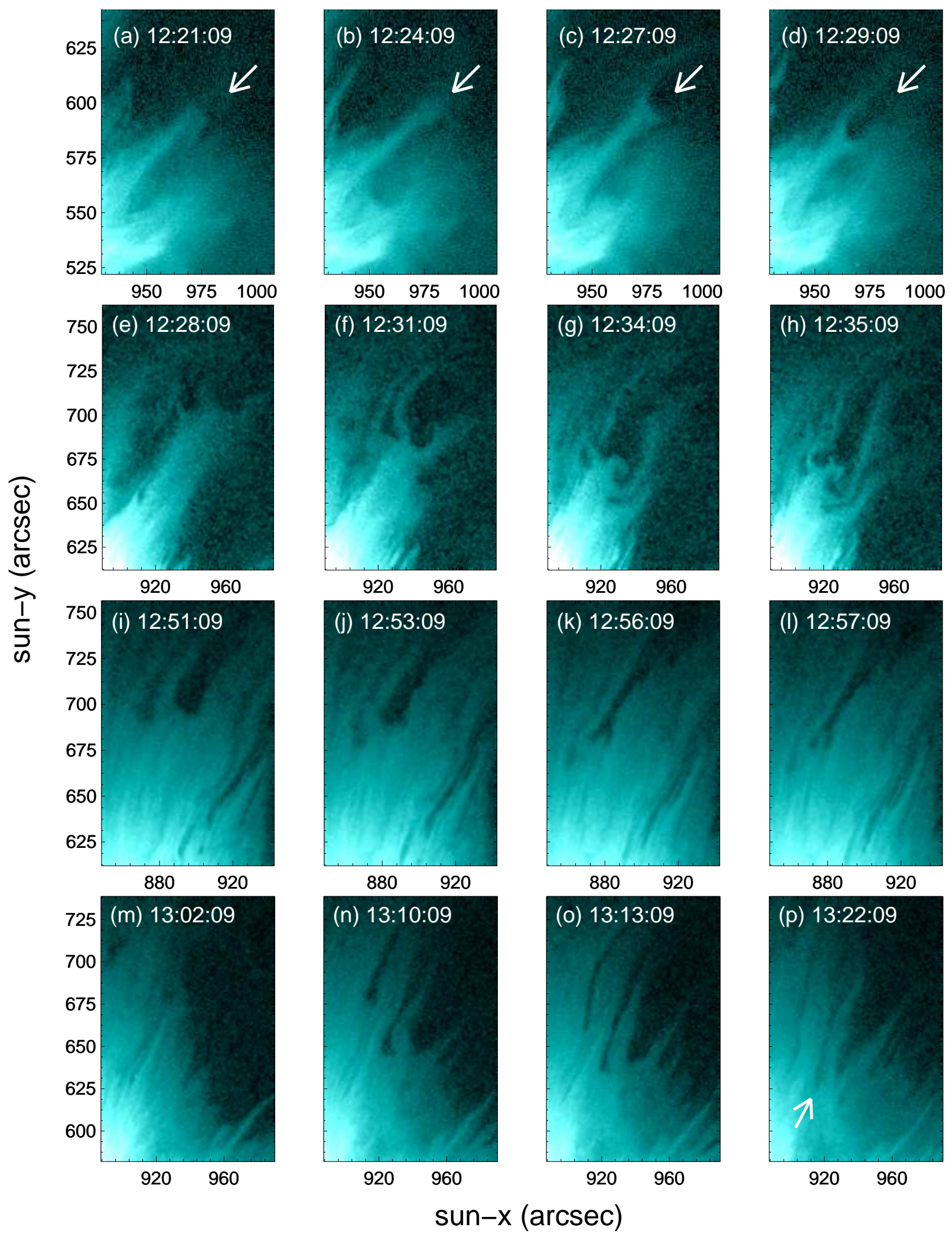

FIG. 2.- SDO/AIA $131 \AA$ channel images showing the evolution of SADs in the supra-arcade fan of 2011 October 22 . (a)-(d) Appearance of a SAD at the top of a growing fan spike. (e)-(h) The head of this large SAD develops prongs at the side of the head. (i)-(l) The head of a smaller SAD also splits in two. (m)-(p) Long-lasting SADs that fade above the arcade with no indication of brightening at their heads. The white arrows in the top row point to a growing fan spike where a SAD appeared at its apex. The white arrow in (p) points to the head of a fading SAD. 


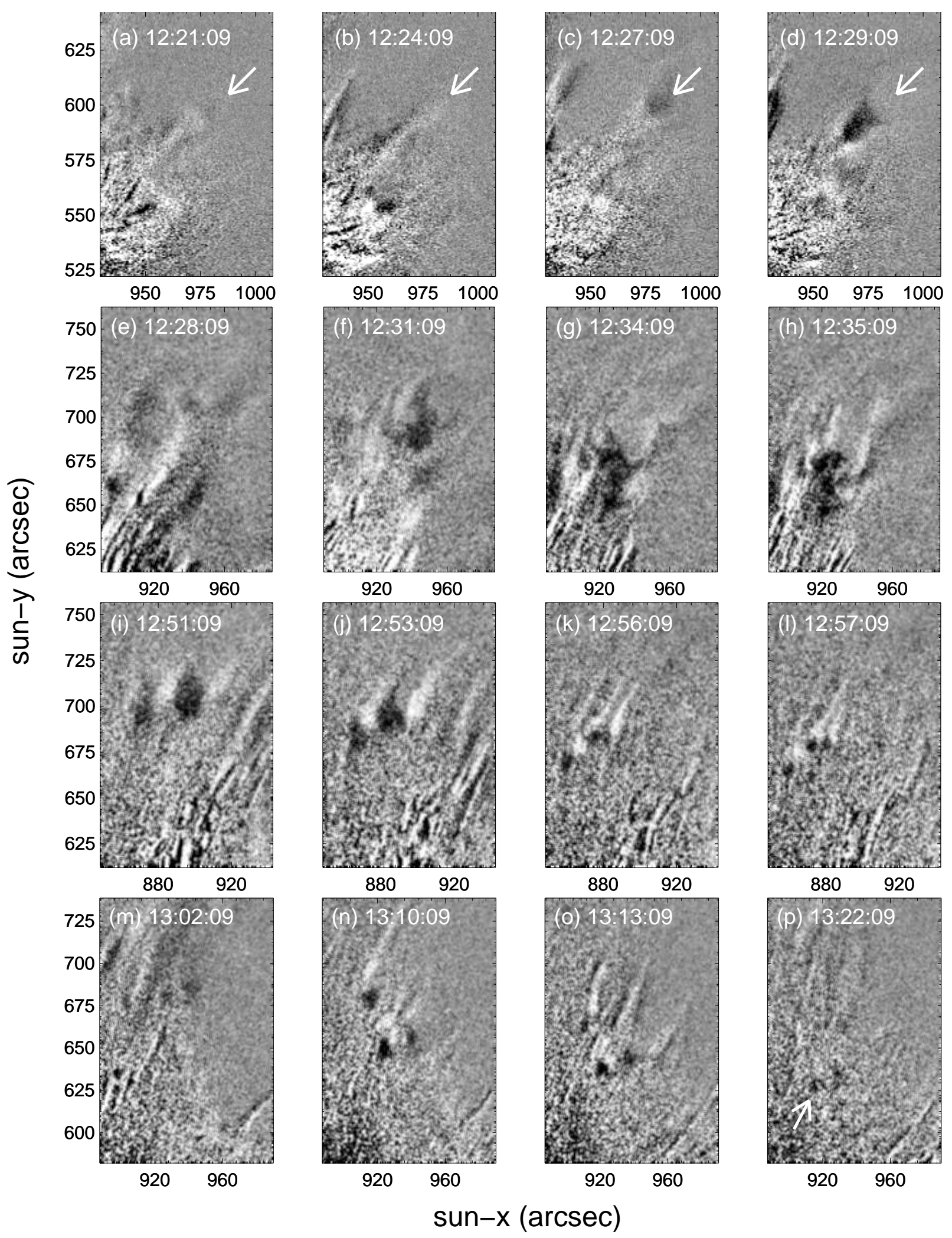

FIG. 3.- SDO/AIA $131 \AA$ difference images at the same time as the images in Figure 2 In each case, the subtracted image was taken 2 min earlier. 
in Figure 4(b) and (d), appeared at the top of one of the fan spikes. This was a very narrow spike and the SAD appeared almost exactly at its apex, suggesting that the presence of the spike may be an important aspect of the SAD's formation. This however requires a detailed study of the statistics of SADs and spikes. It is also possible that the SAD forms higher in the corona and falls onto the fan spike.

In the second example, Figure $4(\mathrm{f})$ and $(\mathrm{h})$, the main fan spike moved sideways, pushed by downflowing plasma. Liu (2013) has shown that this downflow appeared to be related to a slowly retracting SADL underneath. There is however considerable line-of-sight confusion because emission is integrated along the whole length of the arcade, so it is not clear how the SAD and SADL are related. The distance between the SAD and SADL is rather large for the SAD to be the wake of the SADL, so Liu (2013) suggested that the SAD is a twisted flux rope contracting onto the arcade.

\subsection{January 19}

This event gives a less confused view of SADs forming below an edge-on current sheet because the supra-arcade fan is projected against the disk, and viewed at a slight angle so that the northern part has the dark disk as background while the rest is superimposed on the bright flare arcade. The STEREO images of this event show that there were many SADs along the whole arcade (e.g. Figure 1(f)). As shown in the online movie, movie_12jan19, individual SADs can clearly be picked out in AIA images at the northern end of the arcade. Again several SADs are seen at the top of fan spikes. One example is shown in Figure 5. Initially the fan spike, indicated with a white arrow, grows upward with velocity about $50 \mathrm{~km} \mathrm{~s}^{-1}$. The growth of the spike is most visible in the difference images. In Figure 5 (g) the top of the spike is white, indicating increased emission at the top of the spike just before the SAD appears (Figure $5(\mathrm{~h})$ ).

The advantage of this event over the previous two discussed, is that the relationship between SADLs and SADs can be seen much more clearly. SADLs can be unambiguously identified since they appear broad-side rather than edge-on, and there is less line-of-sight confusion because one is only looking through a small part of the arcade not the whole thing. In Figures 5 and 6 . SADLs are indicated with red arrows. Figure 5 shows a SADL that appeared at about the same time and about $25^{\prime \prime}$ below a SAD. There may be a connection between the two because the difference image suggests that the SADL appears at the base of the spike when the SAD appeared at its top. This is similar to the association between the SAD and SADL recorded by Liu (2013) in the 2012 July 19 event. In the second example, shown in Figure 6, the difference images show no connection between the two, and the SAD is descending faster than the SADL. There is also no bright $131 \AA$ emission at the head of the SAD. Several other examples of SAD and SADL evolution can be seen in the movie, movie_12jan19rd. There are both connected and separate SADs and SADLs. In the movie, SADs generally appear above the height where SADLs are seen.

3. DISCUSSION
By looking at the appearance of SADs in the supraarcade fans of three eruptive flares with different orientations of their arcade axes, we find that: (i) the heads of SADs evolve, sometimes splitting into narrower SADs; (ii) a retracting loop, SADL, is sometimes seen below a SAD but there is no strong evidence in our datasets that SADs and SADLs are directly connected; (iii) several SADs appear at the top of rising spikes; (iv) SADs do not necessarily have bright emission at their heads. We also note two other features that have been much discussed and poorly understood: (i) the relatively long lifetime of narrow SADs and (ii) their slow downward velocity, typically $25-50 \mathrm{~km} \mathrm{~s}^{-1}$, as measured from EUV images.

As discussed in Guo et al. (2014), these phenomena can be naturally explained in the context of instabilities in the exhaust of a reconnection jet. Figure 7(a) illustrates the configuration. Following the CSHKP model of eruptive flares (Carmichael 1964; Sturrock 1966; Hirayama 1974; Kopp \& Pneuman 1976), a current sheet forms behind a coronal mass ejection (CME). The core of the CME is usually an elongated filament, so the eruption generates a long, irregular current sheet behind the erupting filament. Reconnection along the current sheet leads to outflows and the formation of an arcade into which plasma continues to accumulate leading to a high density region below the outflowing jet. The outflows occur along the length of the current sheet and create a broad jet of low density, high-speed plasma. When this plasma encounters the higher density plasma that has filled the region between the jet head and the arcade, the interface will be unstable to modes of the RayleighTaylor type (including interchange/ballooning modes, if the plasma conditions allow). A snapshot of the density, temperature and corresponding $131 \AA$ intensity of plasma along the interface, from one of the simulation runs described in Guo et al. (2014) is shown in Figure 7(b)-(d). As can be seen the instability results in fingers of high temperature and low density penetrating into the underlying higher density plasma. The low density fingers, which are part of the reconnection outflow, are threaded by reconnected flux with footpoints rooted in opposite polarities on the solar surface. The difference between the interpretation of SADs presented here and the loop picture (Linton \& Longcope 2006; Savage et al. 2012b; Scott et al. 2013) is that here the SADs are due to an instability in the reconnection outflow whereas in the loop model the SADs are a consequence of retracting loops. It may be that as more of the reconnected flux piles up towards the top of the arcade, chromospheric evaporation causes the head of SADs to fill with plasma, and thus create the SADLs. To simulate this would require more realistic boundary conditions and additional physics. These snapshots were chosen because they show that dark tadpole-shaped structures (SADs) appear at the apex of the high density plumes (spikes), as seen in the observations. The most obvious discrepancy with observation is that the ratio between the finger separation to their length is smaller than typically seen in SADs. In practice, the separation is probably governed by inhomogeneities in the arcade fan, the strength of the magnetic field in the Y-direction, and the reconnection process which may be subject to two-fluid or kinetic ef- 

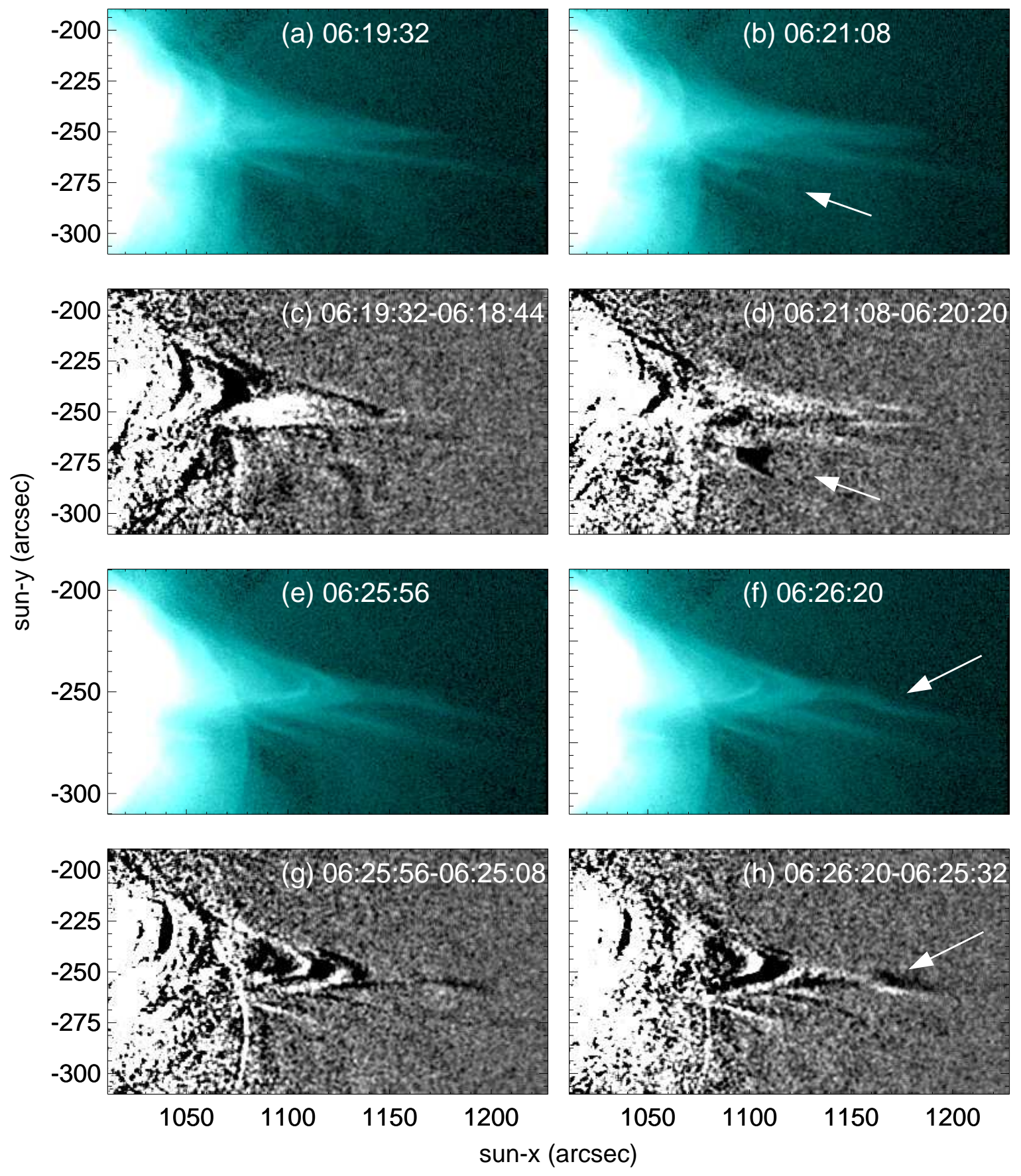

FIG. 4.- SDO/AIA $131 \AA$ intensity and difference images showing the evolution of SADs in the supra-arcade fan of 2012 July 19 . (a)-(b) Formation of a SAD (white arrow) at the top of a fan spike above a cusp arcade. (c)-(d) Corresponding difference images. (e)-(f) SAD formation below the main current sheet (white arrow). (g)-(h) Corresponding difference images.

fects outside of the scope of the MHD model. Another strong point of the model is that it explains the low planeof-sky velocity of SADs seen in EUV images, and their relatively long lifetime.

Other explanations for SADs, in particular the retracting loop model, may be configured to reproduce the splitting at the heads of the SADs if one considers multiple loops, and the lack of $131 \AA$ brightening at their head by arguing that it is outside the bandpass of the observations, or that poor contrast at the top of the fan hides the SADL/shock emission. We hope that the aspects of SAD evolution highlighted here will motivate further modelling, especially of the retracting loop models to test these hypotheses.

The instability model explains the principle features of SADs discussed here, and provides a coherent picture of reconnection outflows, the formation of arcades, supraarcade fans, and SADs. We conclude therefore that SADs appear due to instability at the interface where a fast, low density, reconnection jet encounters higher 


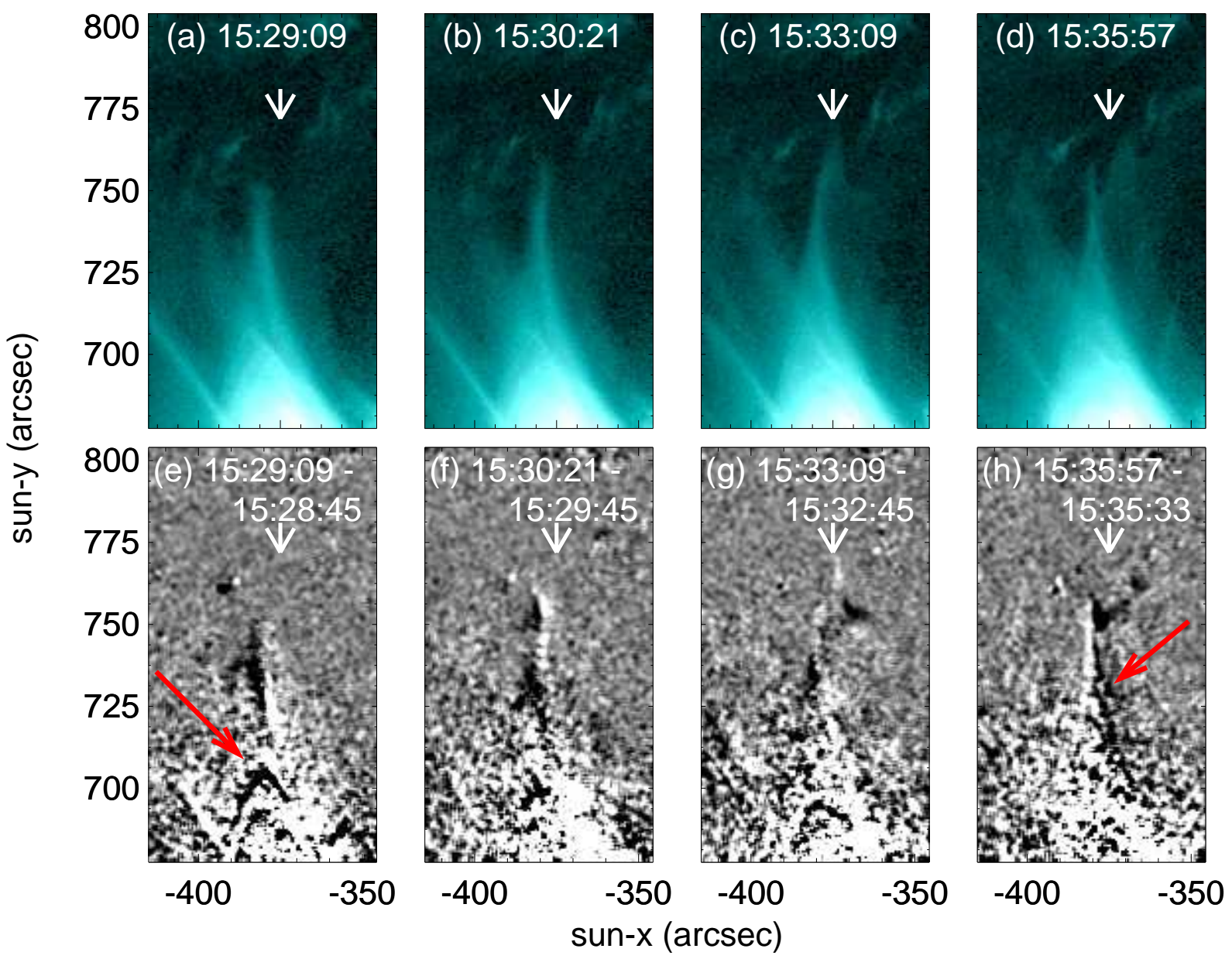

FIG. 5.- SDO/AIA $131 \AA$ intensity and difference images showing the evolution of SADs in the supra-arcade fan of 2012 January 19. (a)-(d) Formation of a SAD (white arrow) at the top of a fan spike above a cusp arcade. (e)-(h) Corresponding difference images. The white arrows indicate the fan spike where the SAD formed. The red arrows indicate SADLs.

density supra-arcade fan plasma.

We thank the referees for constructive comments. This work is supported by the Department of Energy, Grant No. DE-FG02-14-07ER46372, under the auspices of the
Center for Integrated Computation and Analysis of Reconnection and Turbulence (CICART), the National Science Foundation, Grant No. PHY-0215581 (PFC: Center for Magnetic Self-Organization in Laboratory and Astrophysical Plasmas), NASA Grant Nos. NNX09AJ86G and NNX10AC04G, and NSF Grant Nos. ATM-0802727, ATM-090315 and AGS-0962698.

\section{REFERENCES}

Asai, A., Yokoyama, T., Shimojo, M., \& Shibata, K. 2004, ApJ Lett., 605, L77

Berger, T., Testa, P., Hillier, A., et al. 2011, Nat, 472, 197

Berger, T. E., Slater, G., Hurlburt, N., et al. 2010, ApJ, 716, 1288

Carmichael, H. 1964, in The Physics of Solar Flares, ed. W. N. Hess (NASA SP-50; Washington, DC: NASA), 451

Cassak, P. A., Drake, J. F., Gosling, J. T., et al. 2013, ApJ, 775, L14

Cécere, M., Schneiter, M., Costa, A., Elaskar, S., \& Maglione, S. 2012, ApJ, 759, 79

Ciaravella, A. \& Raymond, J. C. 2008, ApJ, 686, 1372

Costa, A., Elaskar, S., Fernández, C. A., \& Martínez, G. 2009 MNRAS, 400, L85

Doschek, G. A., McKenzie, D. E., \& Warren, H. P. 2014, ApJ, 788,26

Guidoni, S. E. \& Longcope, D. W. 2011, ApJ, 730, 90

Guo, L.-J., Bhattacharjee, A., Huang, Y.-M., \& Innes, D. E. 2014, ArXiv e-prints

Hanneman, W. J. \& Reeves, K. K. 2014, ApJ, 786, 95

Hillier, A., Isobe, H., Shibata, K., \& Berger, T. 2012, ApJ, 756, 110
Hirayama, T. 1974, Sol. Phys., 34, 323

Howard, R. A., Moses, J. D., Vourlidas, A., et al. 2008, Space Science Reviews, 136, 67

Innes, D. E., Cameron, R. H., Fletcher, L., Inhester, B., \& Solanki, S. K. 2012, A\&A, 540, L10

Innes, D. E., McKenzie, D. E., \& Wang, T. J. 2003a, Sol. Phys., 217,267

Innes, D. E., McKenzie, D. E., \& Wang, T. J. 2003b, Sol. Phys., 217,247

Khan, J. I., Bain, H. M., \& Fletcher, L. 2007, A\&A, 475, 333

Ko, Y.-K., Raymond, J. C., Lin, J., et al. 2003, ApJ, 594, 1068

Kopp, R. A. \& Pneuman, G. W. 1976, Sol. Phys., 50, 85

Lemen, J. R., Title, A. M., Akin, D. J., et al. 2012, Sol. Phys., 275,17

Linton, M. G. \& Longcope, D. W. 2006, ApJ, 642, 1177

Liu, R. 2013, MNRAS, 434, 1309

Liu, W., Chen, Q., \& Petrosian, V. 2013, ApJ, 767, 168

Maglione, L. S., Schneiter, E. M., Costa, A., \& Elaskar, S. 2011, A\&A, 527, L5

McKenzie, D. E. 2000, Sol. Phys., 195, 381

McKenzie, D. E. 2013, ApJ, 766, 39 


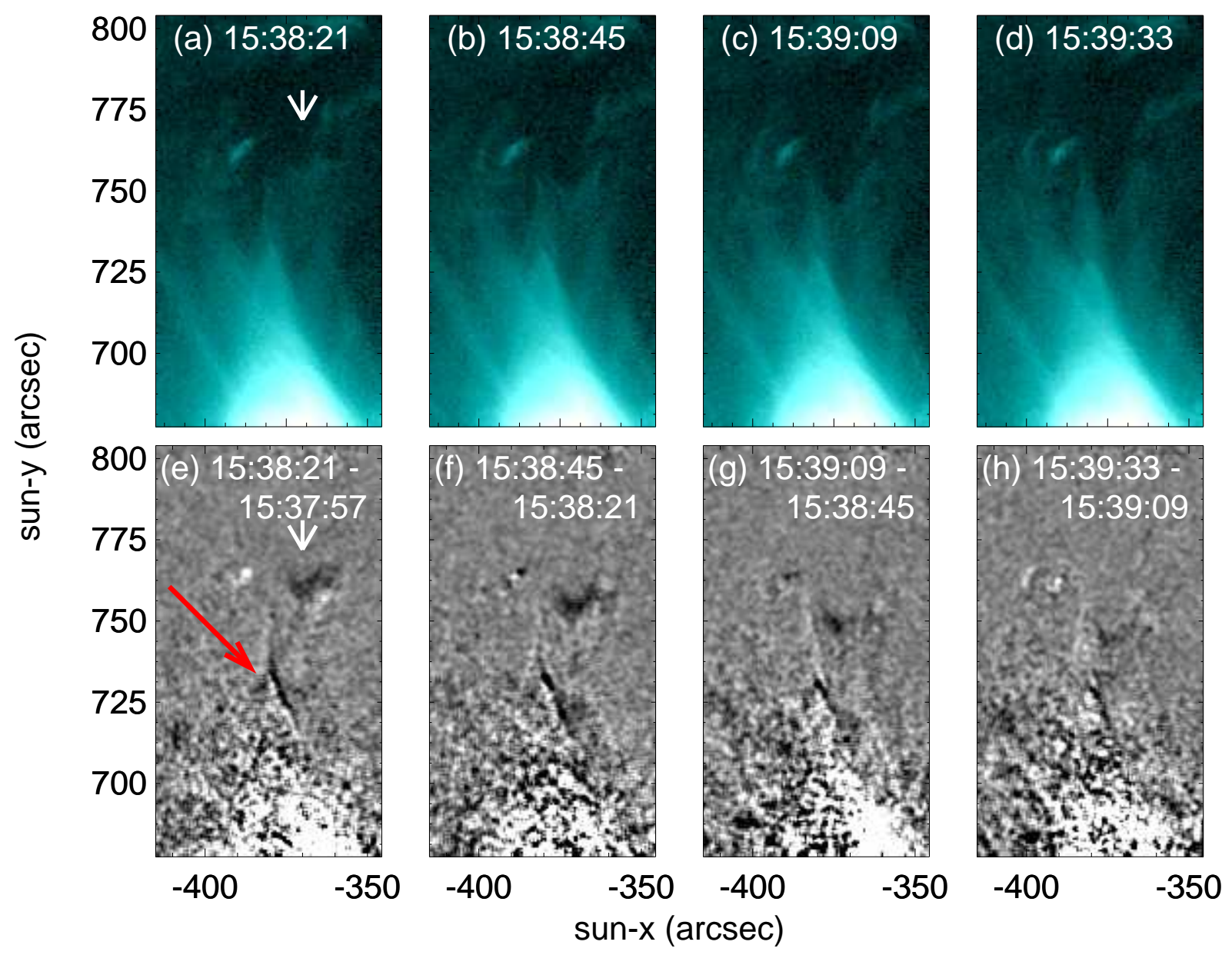

FIG. 6.- SDO/AIA $131 \AA$ intensity and difference images showing the evolution of SADs in the supra-arcade fan of 2012 January 19. (a)-(d) SAD (white arrow) with no SADL at its head. (e)-(h) Corresponding difference images. The red arrow indicates the nearest SADLs.

McKenzie, D. E. \& Hudson, H. S. 1999, ApJ Lett., 519, L93

Savage, S. L., Holman, G., Reeves, K. K., et al. 2012a, ApJ, 754, 13

Savage, S. L. \& McKenzie, D. E. 2011, ApJ, 730, 98

Savage, S. L., McKenzie, D. E., \& Reeves, K. K. 2012b, ApJ, 747, L40

Savage, S. L., McKenzie, D. E., Reeves, K. K., Forbes, T. G., \& Longcope, D. W. 2010, ApJ, 722, 329

Scott, R. B., Longcope, D. W., \& McKenzie, D. E. 2013, ApJ, 776,54
Sturrock, P. A. 1966, nature, 211, 695

TanDokoro, R. \& Fujimoto, M. 2005, Geophys. Res. Lett., 32, 23102

Švestka, Z., Fárník, F., Hudson, H. S., \& Hick, P. 1998, Sol. Phys., 182, 179

Verwichte, E., Nakariakov, V. M., \& Cooper, F. C. 2005, A\&A, 430, L65

Warren, H. P., O'Brien, C. M., \& Sheeley, Jr., N. R. 2011, ApJ, 742,92 

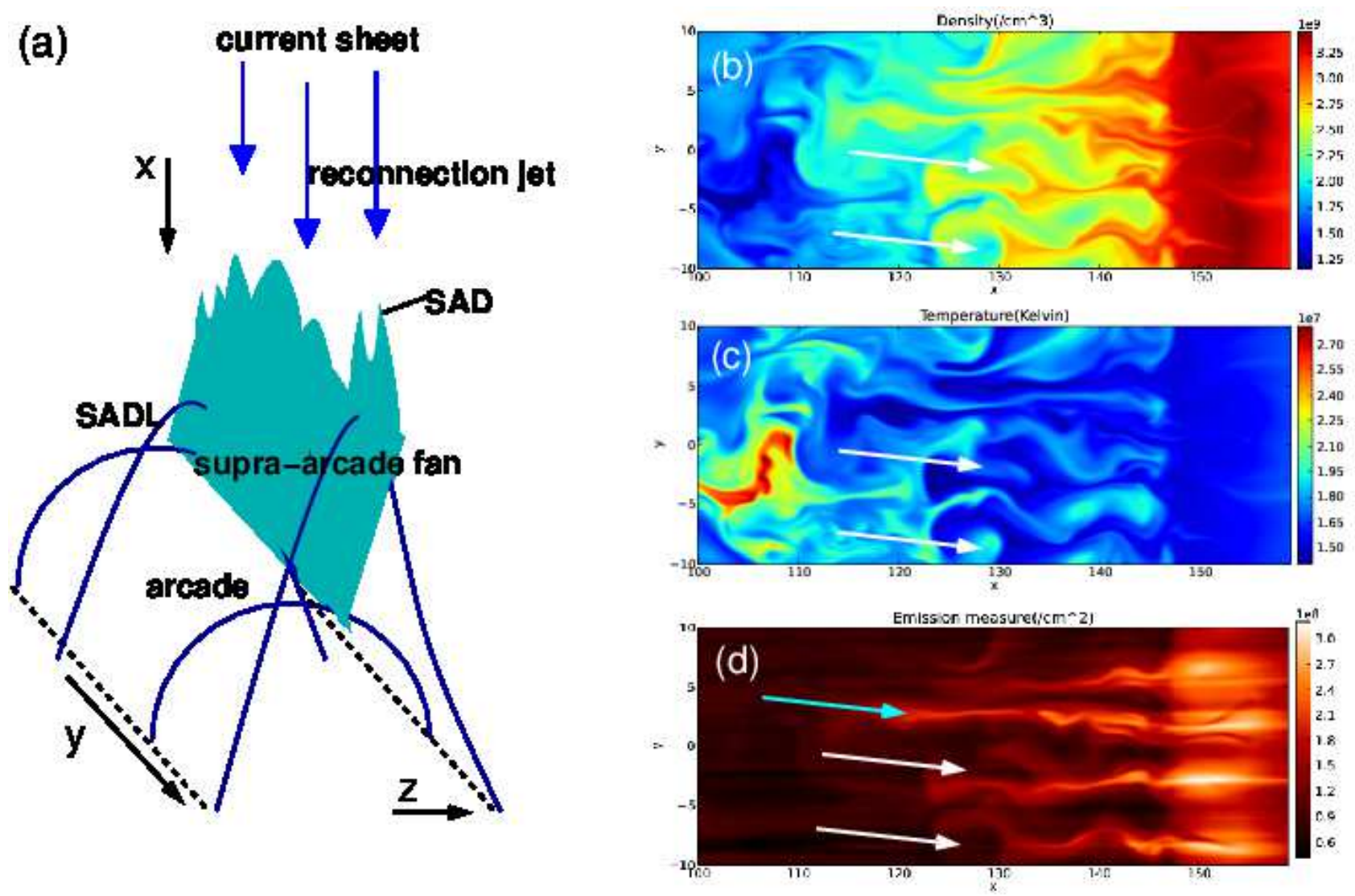

FIG. 7.- (a) Sketch of a supra-arcade showing the configuration of the reconnection jet and supra-arcade fan. (b)-(d) Simulations of instabilities at the head of a reconnection jet: (b) density; (c) temperature; (d) emissivity in the AIA 131 A channel. Temperature and density are shown along the plane passing through the center of the current sheet $(\mathrm{Z}=0)$, and emissivity is integrated across the $\mathrm{Z}$-direction. The directions X, Y, Z are indicated in (a). The white arrows in (b)-(d) point to downflows forming on top of rising high-density fingers. The turquoise arrow in (d) points to a fan spike. 